\section{Cockroach allergy: risk factor for asthma severity}

Dear Editor,

Until the mid-1970s aeroallergens were regarded as triggers of asthma attacks in individuals whose lungs were "genetically irritable." More recently, however, the role of allergen exposure in the development of asthma has been more clearly defined, and it is believed to have three basic functions: a) exposure to some specific allergen(s), especially in early life, makes genetically susceptible individuals develop hypersensitivity; b) In these individuals, continuous exposure to the allergen(s) increases the risk for bronchial hyperresponsiveness (BHR) and; c) most individuals with BHR have repeated episodes of airflow obstruction, which may be triggered by several factors other than allergens, such as cold air, tobacco smoke, stress, among others.

Thus, the relationship between exposure to allergens and development of asthma seems to be restricted only to individuals who developed type I hypersensitivity, i.e., atopic individuals. Therefore, it is quite likely that: a) more than a direct effect on lungs, the action of allergenic proteins may result from immediate and late allergic reactions and b) even though they act as triggers of asthma attacks, allergens per se are the main cause of inflammation that coexists with asthma in individuals who are genetically susceptible to atopic disease. ${ }^{1}$

For some decades, it has been known that household dust and mites may cause atopic sensitization, with the development of different clinical symptoms. Among those aeroallergens that play a role in the pathogenesis of respiratory allergies, one should pay special attention to those produced by fungi, cockroaches, pollen, and pet dander.

Cockroach infestation is a universal phenomenon, but, although there are nearly 3,500 cockroach species all over the world, few of them inhabit households. Sensitization to cockroach allergens, a problem that predominantly affects urban residents, has been more deeply implicated in the etiology of asthma, mainly regarding more severe clinical symptoms among socioeconomically underprivileged patients. ${ }^{2}$

In a study carried out in São Paulo, which used skin tests to determine atopic sensitization, we observed the remarkable presence of sensitization to cockroach allergens (Blattella germanica and Periplaneta americana) in a group of asthmatic adolescents ( 22.3 and $16.5 \%$, respectively) compared to non-asthmatic individuals (controls) ( 0 and $0 \%$, respectively). In the asthmatic group, the rate of BHR was significantly higher among sensitized adolescents, compared to nonsensitized ones. These data were corroborated by the presence of more severe asthma (higher frequency of wheezing episodes and speech disorders due to wheezing in the past year) among sensitized asthmatics. ${ }^{3}$

The diagnosis of respiratory allergies is based on the correlation between clinical history and physical examination specific to allergies, in addition to diagnostic test results. IgE is the major antibody class involved in type I hypersensitivity. Thus, the in vivo and in vitro tests for the diagnosis of allergy are used to detect free IgE or cell membrane-bound IgE.

The study by Lopes et al. ${ }^{4}$ is of utmost importance, since it shows the key role of cockroach allergens in atopic sensitization in our setting and presents important facts about the tests used for its diagnosis. A previous study found $32.8 \%$ of positive results for cockroach-specific $\mathrm{IgE}^{5}$ among Brazilian atopic children treated at divisions of Pediatric Allergy.

We congratulate the authors on the choice of the topic, on their careful observations, and on the extremely appropriate way by which they addressed the limitations and controversies regarding the methods used for allergen-specific IgE determination: in vivo (skin tests) and in vitro (specific IgE measurement).

The authors adequately discuss a web of factors that may influence the results of these tests and that should always be taken into account. Among these factors are the quality and power of extracts, the techniques used, the possibility of cross-reactions and the accuracy of interpretation.

No less important was the discussion about total IgE determination, which, as the authors stated, is often requested, but has a too limited and arguable application in the diagnosis of allergies.

It should be underscored that it is impossible to diagnose "allergy" based solely on the results of laboratory (in vivo or in vitro) tests for specific IgE. The diagnosis must be hinged on the correlation between clinical history, physical examination, and test results.

Despite the improvements in, and the growing importance of, in vitro tests as auxiliary tools in the diagnosis of type I allergies in the last few decades, in vivo skin testing is the most adequate method, from a clinical standpoint, for the assessment of atopic sensitization.

For this purpose, the qualitative determination of the allergens to be used in the batteries of tests should always take the regional characteristics of aeroallergen prevalence into consideration, i.e., potential and known allergen exposure for the region and area of the country where the patient lives. ${ }^{6}$ 


\section{References}

1. Platts-Mills TAE. How environment affects patients with allergic disease: indoor allergen and asthma. Ann Allergy. 1994;72: 381-4.

2. Rosenstreich DL, Eggleston P, Kattan M, Baker D, Slavin RG, Gergen $P$, et al. The role of cockroach allergy and exposure to cockroach allergen in causing morbidity among inner-city children with asthma. N Engl J Med. 1997;336:1356-63.

3. Solé D, Camelo-Nunes IC, Wandalsen GF, Chacon K, Naspitz C. Sensitization to cockroach as a marker of asthma severity among probable asthmatic adolescents identified by the International Study of Asthma and Allergies in Childhood (ISAAC) protocol. J Allergy Clin Immunol. 2002;109:S36-37.

4. Lopes MIL, Miranda PJ, Sarinho E. Use of the skin prick test and specific immunoglobulin $\mathrm{E}$ for the diagnosis of cockroach allergy. J Pediatr (Rio J). 2006;82:204-9.

5. Naspitz CK, Solé D, Jacob CA, Sarinho E, Soares FJP, Dantas V et al. Sensitization to inhalant and food allergens in Brazilian atopic children by in vitro total and specific IgE assay. Allergy Project - PROAL. J Pediatr (Rio J). 2004;80:203-10.

6. Comissão de Testes, Imunoterapia e Padronização de Antígenos. Testes cutâneos em alergia. Rev Bras Alerg Imunopatol. 2000;23: 134-6.

doi:10.2223/JPED.1540

\section{Inês C. Camelo-Nunes}

Disciplina de Alergia, Imunologia Clínica e Reumatologia, Departamento de Pediatria, Universidade Federal de São Paulo Escola Paulista de Medicina (UNIFESP-EPM), São Paulo, SP, Brasil.

\section{Dirceu Solé}

Disciplina de Alergia, Imunologia Clínica e Reumatologia, Departamento de Pediatria, UNIFESP-EPM, São Paulo, SP, Brasil.

\section{Authors' reply}

Dear Editor,

We greatly appreciate the comments by Camelo-Nunes and Solé about our article on cockroach sensitization. ${ }^{1}$ Cockroach allergy has been known for over 40 years and, since then, studies have been carried out worldwide, showing the association between the development of specific IgE antibodies against cockroach allergens and asthma. Despite this fact, even in the USA, where most of the research on this topic is conducted, parents or surrogates of asthmatic children associate the cause of asthma attacks with tobacco smoke, fungi, dust, and inhaled irritants, but not with cockroaches. ${ }^{2}$ As exposure is chronic, and often goes unnoticed by household members because cockroaches hide from view most of the time, living in a parallel universe, there is usually no reference to the development of symptoms as a result of exposure to cockroaches.

Some studies have suggested that asthmatic individuals with cockroach sensitization belong to a distinct subgroup characterized by a more severe course of the disease. In a study undertaken in the city of Curitiba, Brazil, which included 303 atopic asthmatics aged 7 to 14 years, the frequency of sensitization assessed by skin tests using a mixed extract of $B$. germanica and $P$. americana amounted to $19.7 \%$ in children with mild asthma, to $30.1 \%$ in children with moderate asthma and to $40.7 \%$ in those with severe asthma, with a statistically significant difference. ${ }^{3}$ Since only $7 \%$ of our asthmatic patients had severe asthma, we categorized them into two groups in order to assess sensitization according to disease severity: mild asthma (46\%) and moderate/severe asthma (54\%). Sensitization to $B$. germanica and/or $P$. americana among patients with moderate/severe asthma in relation to those patients with mild asthma amounted respectively to 41.5 versus $25.7 \%$ on the skin tests and to 51.2 versus $34.3 \%$ in the specific IgE measurement. No statistically significant difference was observed.

We may not have found a significant difference due to the sample size used, which was not calculated specifically for this purpose, or due to the small number of patients classified as having severe asthma. Although the samples were collected from an allergic disease outpatient clinic, approximately $50 \%$ of patients had mild asthma, associated with more severe symptoms of allergic rhinitis. This may explain the high rate of cockroach sensitization in this group $(25.7 \%$ on skin tests and $34.3 \%$ in the specific IgE measurement).

Since the levels of allergen exposure in patients' households were not determined, the analysis of asthma severity was limited to the assessment of cockroach sensitization, which, in isolation, is not associated with more severe disease. ${ }^{4}$ It was not possible to quantify exposure in households through monoclonal antibody assays. Therefore, data about exposure were obtained from the subjective impression of parents or surrogates about the presence of cockroaches in the household in the last 6 months. However, visual impression is not a good marker of allergen exposure, as allergens are detected in household dust in up to $48 \%$ of cases in which no signs of infestation exist. 5

In case of a patient with a clinical picture that suggests allergic disease, clinical history and physical examination often have to be complemented with laboratory tests. Symptoms are usually common to other morbidities, and allergens from different sources may be involved. This is especially complex when perennial symptoms are considered, i.e., when there is no clear association between the exposure to a specific allergen and the development of symptoms, in addition to the difficulty surrounding the difference between sensitization and atopic disease, since many individuals with sensitization to inhalant allergens do not present with asthma and/or allergic rhinitis symptoms. ${ }^{6}$

Our study does not provide direct evidence that cockroaches caused asthma among the children and adolescents assessed. From the association between cockroach sensitization, either by the skin test or by specific IgE measurement, and asthma, our conclusion is that this sensitization may be an important risk factor for the disease. Nevertheless, only a more in-depth longitudinal study with a multivariate analysis model including the various risk factors involved will allow determining the actual effect of cockroach sensitization on asthma risk and severity. 


\section{References}

1. Lopes MIL, Miranda PJ, Sarinho E. Use of the skin prick test and specific immunoglobulin $\mathrm{E}$ for the diagnosis of cockroach allergy. J Pediatr (Rio J). 2006;82:204-9.

2. Krieger JW, Song L, Takaro T, Stout J. Asthma and the home environment of low-income urban children: preliminary findings from the Seattle-King County healthy home project. J Urban Health. 2000;77:50-67.

3. Rosário NA, Faria L, Riedi CA, Zulato S. Sensibilização a baratas em crianças asmáticas: relação com a gravidade da doença. Rev Bras Alerg Imunopatol. 1999;22:151-5.

4. Rosenstreich DL, Egglestone $P$, Kattan $M$, Baker D, Slavin RG, Gergen $\mathrm{P}$, et al. The role of cockroach allergen and exposure to cockroach allergen in causing morbidity among inner-city with asthma. N Engl J Med. 1997;336:1356-63.

5. Chew GL, Burge HA, Dockery DW, Muilenberg ML, Weiss ST, Gold DR. Limitations of a home characteristics questionnaire as a predictor of indoor allergens levels. Am J Respir Crit Care Med. 1998; 157:1536-41.

6. Kerkhoff M, Schouten JP, Monchy JGR. The association of sensitization to inhalant allergens with allergy symptoms: the influence of bronchial hyperresponsiveness and blood eosinophil count. Clin Exp Allergy. 2000;30:1387-94.

doi:10.2223/JPED.1545

\section{Maria Isabela L. Lopes}

Mestre, Departamento de Pediatria, Universidade Federal de Pernambuco (UFPE), Recife, PE, Brasil.

\section{Paulo J. Miranda}

Mestre, Departamento de Patologia e Imunopatologia, Laboratório Keizo Asami (LIKA), UFPE, Recife, PE, Brasil.

\section{Emanuel Sarinho}

Doutor, Departamento de Pediatria, Divisão de Alergia e Imunologia, UFPE, Recife, PE, Brasil. therefore, it is necessary to identify patients with IgA deficiency (IgAD) beforehand in order to rule out false-negative results.

Of 361 diabetic patients selected by Araújo et al. ${ }^{1}$, seven (1.9\%) had IgAD.

In a previous study carried out at Hospital de Clínicas of Universidade Federal do Paraná, Brazil, eight out of 149 diabetic children and adolescents screened for CD had IgAD ( $\mathrm{IgA}<5 \mathrm{mg} / \mathrm{dL}$ ), which corresponds to a prevalence of $5.3 \%$. Serum IgA levels were measured by the enzyme-linked immunosorbent assay (ELISA), standardized to determine serum IgA titers below the radial immunodiffusion sensitivity level in the low-concentration plate and turbidimetry. In this same group of diabetic patients, the diagnosis of CD was confirmed in $8.7 \%(13 / 149)$ by anti-endomysial antibody testing and intestinal biopsy. ${ }^{4}$

Liblau et al. ${ }^{5}$ reported that one out of 261 diabetic patients in France had IgAD, a higher prevalence rate than that for the normal French population, which corresponds to $1: 1,400$. In Italy, IgAD was detected in seven of 191 diabetic patients, i.e., a prevalence of $1: 27$, higher than that for the normal Italian pediatric population $(1: 500){ }^{6}$

The prevalence of CD in DM-1 patients, recently assessed by anti-endomysial antibody testing and intestinal biopsy in the state of São Paulo, Brazil, amounted to $4.8 \%$, comparable to the prevalence rate described in U.S. and European studies. ${ }^{7}$

Tanure et al. found a prevalence of $2.6 \%$ for $C D$ in diabetic patients from the Brazilian state of Minas Gerais. ${ }^{8}$ The patients were identified based on the positive results for antigliadin antibodies (AGA), anti-endomysial antibodies and intestinal biopsy. ${ }^{7}$ However, only diabetic patients with positive IgG-AGA and negative IgA-AGA results had their IgA level measured by nephelometry. The 12 patients who were positive only for IgG-AGA had normal serum IgA levels.

We agree that multicenter studies should be conducted in Brazil on the association of CD and DM-1 and that diabetics should be screened for $C D$ on a routine basis. However, due to the higher prevalence of IgAD among diabetic patients, the serum IgA level should be determined before the serological tests for the detection of anti-tTG and anti-endomysial antibodies of the IgA isotype class for the screening of CD. This eliminates false-negative results, by the use of criteria established for IgAD, and by more sensitive methods for IgA measurement, such as ELISA (Table 1).

Table 1 - Prevalence of IgAD and CD in DM-1 patients, according to different studies

\begin{tabular}{lccc}
\hline & DM-1 & IgAD & CD \\
\hline Curitiba, Brazil $^{4}$ & 149 & $8(5.3 \%) *$ & $13(8.7 \%)$ \\
Belo Horizonte, Brazil8 $^{8}$ & 234 & $?$ & $6(2.6 \%)$ \\
São Paulo, Brazil $^{7}$ & 104 & $3(2.9 \%)$ & $5(4.8 \%)$ \\
Recife, Brazil $^{1}$ & 354 & $7(2.0 \%)$ & $37(10.5 \%)$ \\
France $^{5}$ & 261 & $1(0.4 \%)$ & NP \\
Italy $^{6}$ & 191 & $7(3.6 \%)$ & NP
\end{tabular}

$\mathrm{CD}=$ celiac disease $\lg \mathrm{AD}=\lg \mathrm{A}$ deficiency $\mathrm{DM}-1$ = type 1 diabetes mellitus; $\mathrm{NP}=$ not performed.

* Criteria for $\lg A D$ : serum $\lg A<5 \mathrm{mg} / \mathrm{dL}$.
Serological tests for the detection of anti-tTG and antiendomysial antibodies should be reserved for IgA isotypes; entitled "Serum prevalence of celiac disease in children and adolescents with type 1 diabetes mellitus." 1

In most diabetic patients, celiac disease $(C D)$ is insidious and asymptomatic; therefore, serological screening for $C D$ is crucial for an early diagnosis and introduction of appropriate treatment. ${ }^{2}$ In a cross-sectional study, Araújo et al. ${ }^{1}$ found a prevalence of $10.5 \%$ for $C D$ among children and adolescents with type 1 diabetes mellitus (DM-1) using the anti-tissue transglutaminase (anti-tTG) antibody assay, recommended as the test of choice for the initial screening of $C D$ in diabetic patients. $^{3}$ 